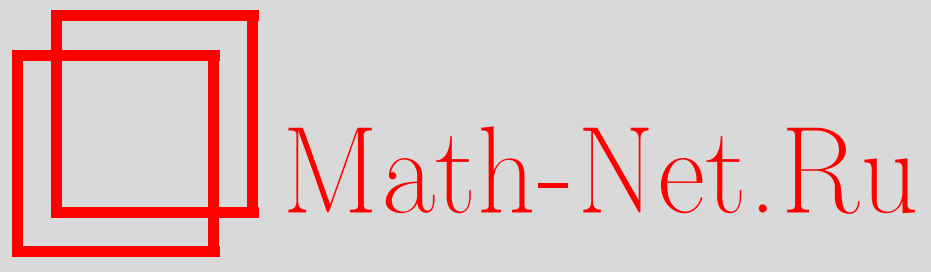

М. И. Зеликин, Геометрия двойного отношения операторов, Матем. сб., 2006, том 197, номер 1, 39-54

DOI: https://doi.org/10.4213/sm1495

Использование Общероссийского математического портала Math-Net.Ru подразумевает, что вы прочитали и согласны с пользовательским соглашением http://www . mathnet.ru/rus/agreement

Параметры загрузки:

IP: 35.173 .219 .12

26 апреля 2023 г., 15:49:40

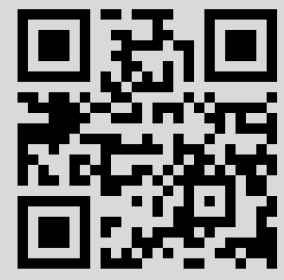




\author{
М. И. Зеликин
}

\title{
Геометрия двойного отношения операторов
}

В работе определяется и изучается двойное отношение операторов, имеющее смысл в том числе и для бесконечномерного грассманова многообразия Сато. Дается гомологическая интерпретация двойного отношения. Вводится матричный и операторный аналог дифференциального оператора Шварца и устанавливается его связь с линейными гамильтоновыми системами и с уравнением Риккати. Эти конструкции имеют целью применение к КП-иерархии (иерархии Кадомцева-Петвиашвили).

Библиография: 12 названий.

\section{§ 1. Грассманиан Сато и КП-иерархия}

Имеется два подхода к изучению интегрируемых систем дифференциальных уравнений - алгебраический и аналитический. Оба подхода в некотором смысле параллельны, и оба приводят к важному геометрическому объекту - бесконечномерному грассманову многообразию, введенному в работах М. Сато [1]. В настоящей статье в основном рассматривается более сложный аналитический вариант. При этом наши конструкции (за исключением теоремы 2) применимы также и к алгебраическому случаю.

При описании алгебраического подхода используются следующие стандартные обозначения. Полем констант $k$ можно считать $\mathbb{R}$ или $\mathbb{C}$. В качестве основного кольца рассматривается кольцо формальных рядов Тейлора $B=k[[x]]=\left\{\sum_{i \geqslant 0} a_{i} x^{i} \mid a_{i} \in k\right\}$. Пусть $E=k[[x]]\left(\left(\partial^{-1}\right)\right)-$ кольцо формальных псевдодифференциальных операторов, снабженное $\partial^{-1}$-адической топологией. Таким образом, $E$ - кольцо формальных рядов Лорана с фильтрацией $V(n)$, соответствующей этому нормированию, т.е. с фильтрацией по степеням главного члена относительно $\partial^{-1}$. Рассмотрим разложение кольца $E$ в прямую сумму $E=E_{+} \oplus E_{-}$, где $E_{+}=B[\partial]$ - кольцо дифференциальных операторов; $E_{-}=B\left[\left[\partial^{-1}\right]\right] \partial^{-1}-$ кольцо псевдодифференциальных операторов отрицательного порядка (операторов типа Вольтерра). Тем самым, для любого $P \in E$ однозначно определено разложение $P=P_{+}+P_{-}$, где $P_{+}-$дифференциальная часть, а $P_{-}-$вольтерровская часть оператора $P$. Разложение бесконечномерного пространства в прямую сумму двух его бесконечномерных подпространств будем называть поляризацией.

Грассманиан Сато GrS есть множество всех замкнутых подпространств $W$ в $E / E x$ таких, что отображение $W \oplus V(1) \rightarrow E / E x$ фредгольмово.

В линейном пространстве $E$ рассмотрим аффинное пространство $E^{\prime}=\partial+E_{-}$ и группу (по умножению) $G=1+E_{-}$. Касательное пространство к ним совпадает с $E_{-}$. Отображение $\xi$ из $G$ в $E^{\prime}$ для $S \in G$ определяется формулой $\xi: S \mapsto L=S \partial S^{-1}$, а отображение $\eta$ из $G$ в $\mathrm{GrS}-$ формулой $\eta: S \mapsto S^{-1} w_{0}$,

Работа выполнена при финансовой поддержке Российского фонда фундаментальных исследований (гранты №№ 04-01-00735, 05-01-22001). 
где $w_{0}$ - некоторое фиксированное подпространство $w_{0} \in \mathrm{GrS}$. Дифференциалы этих отображений в точке $S$ действуют на $A \in E_{-}$по формулам $d \xi: A \mapsto\left[A S^{-1}, L\right]$ и $d \eta: A \mapsto-S^{-1} A$ соответственно.

Известный основной результат [1]-[3] (соответствие Сато) заключается в том, что оператору умножения на $\partial^{-n}$, индуцирующему элемент касательного пространства $\operatorname{Hom}(W, E / W)$ к грассманиану Сато (по которому однозначно восстанавливается $S)$, отвечает в пространстве $E^{\prime}$ коммутатор $\left[L_{+}^{n}, L\right]$, т.е. правая часть иерархии Кадомцева-Петвиашвили (КП-иерархия). Если фиксировать $n$, то оператор $\partial^{-n}: E \rightarrow E$ определяет векторное поле на $\mathrm{GrS}$, соответствующее этому оператору, т.е. правую часть уравнения Риккати (см. [4]). Таким образом, КП-иерархия сопоставляется со счетной полисистемой взаимно коммутирующих векторных полей, отвечающих операторным уравнениям Риккати на грассманиане Сато. Аналогичная теория для случая некоммутативного кольца констант развивалась в [5], а для случая многомерного $x-$ в работе [6].

При аналитическом подходе рассматривается гильбертово пространство рядов Лорана $H=\left\{\sum_{-\infty}^{+\infty} a_{n} z^{n} \mid \sum_{-\infty}^{+\infty} a_{n}^{2}<\infty\right\}$, снабженное поляризацией, т.е. разложением в ортогональную сумму двух замкнутых подпространств:

$$
H=H_{+} \oplus H_{-} .
$$

Такое разложение удобно задавать с помощью оператора комплексной структуры, т.е. унитарного оператора $J: H \rightarrow H$, равного + Id на $H_{+}$и - Id на $H_{-}$. Общая ограниченная линейная группа $\mathrm{GL}_{\mathrm{res}}(H)$ определяется как подгруппа $\mathrm{GL}(H)$, состоящая из таких операторов $\mathfrak{A}$, что коммутатор $[J, \mathfrak{A}]$ является оператором Гильберта-Шмидта. Это определение эквивалентно следующему. Если элемент $\mathfrak{A}$ группы $\mathrm{GL}(H)$ записан как $(2 \times 2)$-матрица

$$
\left(\begin{array}{ll}
a & b \\
c & d
\end{array}\right)
$$

по отношению к разложению (1), то $\mathfrak{A}$ лежит в $\mathrm{GL}_{\mathrm{res}}(H)$ тогда и только тогда, когда $b$ и $c$ являются операторами Гильберта-Шмидта. При этом автоматически оказывается, что $a$ и $d$ - операторы Фредгольма; $U_{\mathrm{res}}(H)$ - подгруппа в $\mathrm{GL}_{\mathrm{res}}(H)$, состоящая из унитарных операторов.

Грассманиан Сато GrS в этом случае определяется как множество всех замкнутых подпространств $W$ в $H$ таких, что

1) естественная проекция $W$ на $H_{+}$, т.е. проекция $\mathrm{pr}_{-}: W \rightarrow H_{+}$параллельно подпространству $H_{-}$, является оператором Фредгольма;

2 ) естественная проекция $W$ на $H_{-}$, т.е. проекция $\mathrm{pr}_{+}: W \rightarrow H_{-}$параллельно подпространству $H_{+}$, является оператором Гильберта-Шмидта.

Как известно [2], подгруппа $U_{\text {res }}(H)$ группы $\mathrm{GL}_{\mathrm{res}}(H)$ действует на $\mathrm{GrS}$ транзитивно, причем стабилизатор подпространства $H_{+}$есть $U\left(H_{+}\right) \times U\left(H_{-}\right)$. Тем самым, GrS можно представить как однородное пространство: $\mathrm{GrS}=$ $U_{\text {res }}(H) /\left(U\left(H_{+}\right) \times U\left(H_{-}\right)\right)$. Грассманиан GrS есть бесконечномерное многообразие, моделируемое гильбертовым пространством $J_{2}\left(H_{+}, H_{-}\right)$операторов Гильберта-Шмидта $T: H_{+} \rightarrow H_{-}$, где норма $T \in J_{2}\left(H_{+}, H_{-}\right)$определяется как $\left(\sum_{i}\left\|T e_{i}\right\|^{2}\right)^{1 / 2}$, а $\left\{e_{i}\right\}$ - полный ортонормированный базис в $H_{+}$. Множество таких операторов (точнее, карта на $\mathrm{GrS}$, соответствующая этому множеству) называется большой клеткой и обозначается $\mathrm{GrS}_{+}$. Группа $\mathrm{GL}_{\mathrm{res}}(H)$ действует 
на $\mathrm{GrS}$ преобразованиями Мёбиуса

$$
\left(\begin{array}{ll}
a & b \\
c & d
\end{array}\right) \cdot T=(c+d T)(a+b T)^{-1} .
$$

Здесь разбиение матрицы на блоки соответствует поляризации пространства $H$. Касательное пространство к многообразию $\operatorname{GrS}$ в точке $W$ есть $T_{W}=\operatorname{Hom}(W, H / W)$.

Для изучения КП-иерархии предлагается использовать нижеследующее обобщение двойного отношения [7].

\section{§ 2. Операторное двойное отношение}

Рассмотрим сначала конечномерный случай и подпространства половинной размерности. Пусть $\mathscr{P}_{1}, \mathscr{P}_{2}, \mathscr{P}_{3}, \mathscr{P}_{4}$ - четыре $n$-мерных подпространства в $k^{2 n}$, соответствующие четырем точкам многообразия $\operatorname{Gr}_{n}\left(k^{2 n}\right)$, задаваемые в некоторой фиксированной карте $\mathfrak{U}$ многообразия $\mathrm{Gr}_{n}\left(k^{2 n}\right)(n \times n)$-матрицами $P_{1}, P_{2}, P_{3}, P_{4}$. Предположим, что $\mathscr{P}_{1}, \mathscr{P}_{2}$ и $\mathscr{P}_{3}, \mathscr{P}_{4}$ определяют поляризации $k^{2 n}$, т.е. $\mathscr{P}_{1} \oplus \mathscr{P}_{2}=\mathscr{P}_{3} \oplus \mathscr{P}_{4}=k^{2 n}$. Поляризацию $\mathscr{P}_{i}, \mathscr{P}_{j}$ будем обозначать через $\Pi_{i j}$. Класс матриц $\mathrm{DV}\left(\mathscr{P}_{1}, \mathscr{P}_{2} ; \mathscr{P}_{3}, \mathscr{P}_{4}\right)$, подобных матрице

$$
\left(P_{1}-P_{2}\right)^{-1}\left(P_{2}-P_{3}\right)\left(P_{3}-P_{4}\right)^{-1}\left(P_{4}-P_{1}\right)
$$

(обратные матрицы определены, так как $\Pi_{12}$ и $\Pi_{34}-$ поляризации), является инвариантом упорядоченной четверки точек многообразия Грассмана относительно преобразований Мёбиуса [4]. Он называется матричным двойнъмм отношением.

Операцию проектирования параллельно подпространству $\mathscr{P}_{i}$ будем обозначать через $\pi_{i}$ или цифрой $i$ над стрелкой, обозначающей соответствующее отображение. В том случае, когда $\mathscr{P}_{i}$ и $\mathscr{P}_{j}$ дают поляризацию, образ $\pi_{i}$ в $\mathscr{P}_{j}$ определен однозначно.

ТЕОРема 1. Пусть данъ поляризации $\Pi_{12}$ и $\Pi_{34}$.

Тогда $\mathrm{DV}\left(\mathscr{P}_{1}, \mathscr{P}_{2} ; \mathscr{P}_{3}, \mathscr{P}_{4}\right)$ соответствует оператору сквозного отображения

$$
\mathscr{P}_{1} \stackrel{4}{\longrightarrow} \mathscr{P}_{3} \stackrel{2}{\longrightarrow} \mathscr{P}_{1}
$$

пространства $\mathscr{P}_{1}$ на себя, задаваемого в карте $\mathfrak{U}$ матрицей (2).

ДокАЗАтельство. Пусть $\left(g, P_{3} g\right)$ - проекция элемента $\left(f, P_{1} f\right)$ пространства $\mathscr{P}_{1}$ на $\mathscr{P}_{3}$ параллельно $\mathscr{P}_{4}$. Это означает, что $\left(f-g, P_{1} f-P_{3} g\right) \in \mathscr{P}_{4}$, т.е. $P_{1} f-P_{3} g=P_{4}(f-g)$. Следовательно, $g=\left(P_{4}-P_{3}\right)^{-1}\left(P_{4}-P_{1}\right) f$. Аналогично, проекция элемента $\left(g, P_{3} g\right)$ на $\mathscr{P}_{1}$ параллельно $\mathscr{P}_{2}$ есть $\left(h, P_{1} h\right)$, где $h=\left(P_{2}-P_{1}\right)^{-1}\left(P_{2}-P_{3}\right) g=\left(P_{1}-P_{2}\right)^{-1}\left(P_{2}-P_{3}\right)\left(P_{3}-P_{4}\right)^{-1}\left(P_{4}-P_{1}\right) f$.

ЗАмЕчАниЕ 1. В теории интегрируемых систем [8] $\tau$-функция есть лишь детерминант некоторого операторного двойного отношения; остальные инварианты соответствующего оператора до сих пор не использовались. В работе [8] было доказано менее общее утверждение, относящееся только к детерминанту двойного отношения, совпадающему с $\tau$-функцией.

Теорема 1 позволяет освободиться от предположения половинной размерности и определить двойное отношение для пар поляризаций пространства $k^{n}$. 
Более того, это дает возможность определить (уже не матричное, а операторное) двойное отношение и для бесконечномерного случая. При этом инвариантность операторного двойного отношения относительно действия группы Мёбиуса заложена в самой конструкции в силу линейности операторов проектирования. Заметим, что инвариантно определено само сквозное отображение (3), а его матрица (2) инвариантна с точностью до сопряжения.

ОПРеДЕЛЕниЕ 1. Двойным отношением $\operatorname{DV}\left(\mathscr{P}_{1}, \mathscr{P}_{2} ; \mathscr{P}_{3}, \mathscr{P}_{4}\right)$ четверки подпространств $\mathscr{P}_{1}, \mathscr{P}_{2}, \mathscr{P}_{3}, \mathscr{P}_{4}$, определяющей две поляризации $\Pi_{12}$ и $\Pi_{34}$ пространства $H$, называется сквозное отображение (3).

В дальнейшем будет полезно изменять порядок следования подпространств в данной четверке. Пусть $\mathscr{P}_{1}, \mathscr{P}_{2}, \mathscr{P}_{3}, \mathscr{P}_{4}$ - подпространства линейного пространства $H$. Оператор $\operatorname{DV}\left(\mathscr{P}_{1}, \mathscr{P}_{2} ; \mathscr{P}_{3}, \mathscr{P}_{4}\right)$, определенный в теореме 1 , будем для краткости обозначать $\mathrm{DV}_{12 ; 34}$.

Если $s: B \rightarrow A-$ изоморфизм, то мы будем в дальнейшем отождествлять отображения $f: A \rightarrow A$ и $g: B \rightarrow B$, где $g=s^{-1} f s$.

ЛЕмма 1. Пусть $\Pi_{12}$ и $\Pi_{34}$ - поляризации, и пусть проекиии параллельно $\mathscr{P}_{2}$ и $\mathscr{P}_{4}-$ изоморфизмы пространств $\mathscr{P}_{1}$ и $\mathscr{P}_{3}$.

Тогда $\mathrm{DV}_{12 ; 34}=\mathrm{DV}_{34 ; 12}$.

ДоказАтельство. Рассмотрим отображения

$$
\begin{aligned}
& \mathrm{DV}_{12 ; 34}: \mathscr{P}_{1} \stackrel{4}{\longrightarrow} \mathscr{P}_{3} \stackrel{2}{\longrightarrow} \mathscr{P}_{1}, \\
& \mathrm{DV}_{34 ; 12}: \mathscr{P}_{3} \stackrel{2}{\longrightarrow} \mathscr{P}_{1} \stackrel{4}{\longrightarrow} \mathscr{P}_{3} .
\end{aligned}
$$

Если отождествить $\mathscr{P}_{1}$ и $\mathscr{P}_{3}$ с помощью проекции $\pi_{4}$, то оба рассматриваемые отображения совпадут.

Тем самым, двойное отношение не зависит от порядка пар поляризаций.

Лемма 2. Пусть $\mathscr{P}_{1} \oplus \mathscr{P}_{2}=\mathscr{P}_{3} \oplus \mathscr{P}_{4}=H$, и пусть проекции параллельно $\mathscr{P}_{2}$ и $\mathscr{P}_{4}-$ изоморфизмы пространств $\mathscr{P}_{1}$ и $\mathscr{P}_{3}$.

Тогда $\mathrm{DV}_{12 ; 34}=\mathrm{DV}_{14 ; 32}^{-1}$.

ДокАЗАТЕЛЬСтво. Рассмотрим отображения

$$
\begin{aligned}
& \mathrm{DV}_{12 ; 34}: \mathscr{P}_{1} \stackrel{4}{\longrightarrow} \mathscr{P}_{3} \stackrel{2}{\longrightarrow} \mathscr{P}_{1}, \\
& \mathrm{DV}_{14 ; 32}: \mathscr{P}_{1} \stackrel{2}{\longrightarrow} \mathscr{P}_{3} \stackrel{4}{\longrightarrow} \mathscr{P}_{1} .
\end{aligned}
$$

Имеем $\mathscr{P}_{1} \stackrel{4}{\longrightarrow} \mathscr{P}_{3}=\left(\mathscr{P}_{3} \stackrel{4}{\longrightarrow} \mathscr{P}_{1}\right)^{-1}$ и $\mathscr{P}_{1} \stackrel{2}{\longrightarrow} \mathscr{P}_{3}=\left(\mathscr{P}_{3} \stackrel{2}{\longrightarrow} \mathscr{P}_{1}\right)^{-1}$, откуда и следует утверждение леммы.

Итак, обмен соответствующими друг другу подпространствами между поляризациями (т.е. когда $\Pi_{12}$ и $\Pi_{34}$ переходят в $\Pi_{14}$ и $\Pi_{32}$ ) приводит к замене двойного отношения обратным отображением.

Лемма 3. Пусть все пространства изоморфны и $\mathscr{P}_{1} \oplus \mathscr{P}_{2}=\mathscr{P}_{3} \oplus \mathscr{P}_{4}=$ $\mathscr{P}_{1} \oplus \mathscr{P}_{4}=\mathscr{P}_{3} \oplus \mathscr{P}_{2}=$ H. Тогда $\mathrm{DV}_{12 ; 43}=\mathrm{Id}-\mathrm{DV}_{12 ; 34}$.

ДокАЗАТЕЛЬСтво. Построим образ $h_{1} \in \mathscr{P}_{1}$ при отображении

$$
\mathrm{DV}_{12 ; 43}: \mathscr{P}_{1} \stackrel{3}{\longrightarrow} \mathscr{P}_{4} \stackrel{2}{\longrightarrow} \mathscr{P}_{1} \text {. }
$$


Имеем $\pi_{3} h_{1}=h_{1}+h_{3}$, где $h_{3} \in \mathscr{P}_{3}$, а $h_{1}+h_{3} \in \mathscr{P}_{4}$. Далее, $\pi_{2}\left(h_{1}+h_{3}\right)=$ $h_{1}+h_{3}+h_{2}$, где $h_{2} \in \mathscr{P}_{2}$, а $h_{1}+h_{3}+h_{2} \in \mathscr{P}_{1}$. Перепишем образ $\mathrm{DV}_{12 ; 43}$ в виде $h_{1}+h_{3}+h_{2}=h_{1}-\left(h_{1}-\left(h_{1}+h_{3}+h_{2}\right)\right)$. Поскольку $h_{1}+h_{3} \in \mathscr{P}_{4}$, a $h_{1}-\left(h_{1}+h_{3}\right) \in \mathscr{P}_{3}$, то $h_{1}-\left(h_{1}+h_{3}\right)$ есть образ $h_{1}$ при проекции $\pi_{4}$ на $\mathscr{P}_{3}$. Последующее вычитание $h_{2}$ дает образ проекции $\pi_{2}$ на $\mathscr{P}_{1}$. Поэтому $\left(h_{1}-\left(h_{1}+\right.\right.$ $\left.\left.h_{3}+h_{2}\right)\right)=\mathrm{DV}_{12 ; 34} h_{1}$. Следовательно, $\mathrm{DV}_{12 ; 43}=\mathrm{Id}-\mathrm{DV}_{12 ; 34}$.

Тем самым, изменение порядка подпространств внутри одной из поляризаций приводит к замене двойного отношения DV на Id - DV.

Леммы 1-3 определяют образующие представления группы перестановок из четырех подпространств в группе, порожденной оператором $D=\mathrm{DV}_{12 ; 34}$ и единичным оператором. Ясно, что в случае, когда все пространства изоморфны, можно, комбинируя леммы 1-3, выразить двойное отношение четверки подпространств через двойные отношения тех же подпространств, взятых в любом другом порядке.

Осталось рассмотреть случай, когда подпространства, с которых начинаются (и кончаются) сквозные отображения для сравниваемых двойных отношений, неизоморфны (например, имеют разную размерность). В этом случае соответствующие операторы, определенные на разных подпространствах, различаются лишь наличием прямого слагаемого у большего подпространства, которое отображается тождественно. Сведение к случаю изоморфных подпространств осуществляется следующим образом. Пусть, например, $\operatorname{dim} \mathscr{P}_{2}>$ $\operatorname{dim} \mathscr{P}_{1}$ и мы хотим сравнить $\mathrm{DV}_{12 ; 34}$ и $\mathrm{DV}_{21 ; 43}$. При отображении $\mathrm{DV}_{21 ; 43}$ пространство $\mathscr{P}_{2} \cap \mathscr{P}_{4}$ отображается на себя тождественно, так как его векторы уже лежат как в $\mathscr{P}_{2}$, так и в $\mathscr{P}_{4}$. Пространство $\mathscr{P}_{13}=\mathscr{P}_{1} \oplus \mathscr{P}_{3}$ также является инвариантным, так как проекторы $\pi_{1}$ и $\pi_{3}$ проектируют параллельно этому подпространству. Это пространство инвариантно и относительно $\mathrm{DV}_{12 ; 34}$, так как в нем лежат как $\mathscr{P}_{1}$, так и $\mathscr{P}_{3}$. Таким образом, вопрос сводится к структуре ограничения отображений $\mathrm{DV}_{12 ; 34}$ и $\mathrm{DV}_{21 ; 43}$ на $\mathscr{P}_{13}$. В ситуации общего положения пересечение пространств $\mathscr{P}_{2}$ и $\mathscr{P}_{4}$ с $\mathscr{P}_{13}$ имеют ту же размерность, что и $\mathscr{P}_{1}$, и далее можно применять леммы $1-3$ в пространстве $\mathscr{P}_{13}$ :

$$
\mathrm{DV}_{21 ; 43}=\mathrm{Id}-\mathrm{DV}_{21 ; 34}=\mathrm{Id}-\mathrm{DV}_{34 ; 21}=\mathrm{DV}_{34 ; 12}=\mathrm{DV}_{12 ; 34}
$$

\footnotetext{
Через $\mathscr{P} \perp$ будем обозначать ортогональное дополнение к подпространству $\mathscr{P}$.
}

ОПРЕДЕЛЕНИЕ 2. Под двойственным грассманианом $\widetilde{\operatorname{Gr}}(H)$ к грассманиану $\operatorname{Gr}(H)$ будем понимать совокупность $\left\{\mathscr{P}^{\perp}\right\}$ ортогональных дополнений к подпространствам $\mathscr{P} \in \mathrm{Gr}(H)$.

ЗАмЕчАНИЕ 2. Из приведенного анализа ясно, что правильное обобщение двойного отношения состоит в том, чтобы брать две точки из одного грассманиана, а две другие точки - из двойственного к нему грассманиана для того, чтобы соответствующие пары подпространств образовывали поляризации. В конечномерном случае понятие двойственного грассманиана ясно; в случае же грассманиана Сато его связь с общей ограниченной линейной группой $\mathrm{GL}_{\mathrm{res}}$ требует уточнений, которые будут даны ниже в утверждении 1.

Докажем теперь, что операторное двойное отношение определяет операторнозначный коцикл по дополнениям двух данных изоморфных подпространств до прямой суммы. 
Лемма 4. Пусть даны два подпространства $\mathscr{P}_{i}, i=1,2$, переводимъе друг в друга с помощъю оператора, принадлежащего $U_{\mathrm{res}}$, и три их дополнения до прямой суммы $\mathscr{Q}_{j}, j=1,2,3$ (m.e. $\mathscr{P}_{i} \oplus \mathscr{Q}_{j}=H, i=1,2, j=1,2,3$ ).

Тогда

$$
\operatorname{DV}\left(\mathscr{P}_{1} \mathscr{Q}_{1} ; \mathscr{P}_{2} \mathscr{Q}_{2}\right) \operatorname{DV}\left(\mathscr{P}_{1} \mathscr{Q}_{3} ; \mathscr{P}_{2} \mathscr{Q}_{1}\right) \operatorname{DV}\left(\mathscr{P}_{1} \mathscr{Q}_{2} ; \mathscr{P}_{2} \mathscr{Q}_{3}\right)=\mathrm{Id}
$$

т.е. произведение этих трех операторов есть тождественное отображение.

ДокАЗАТЕЛЬСтво. Рассмотрим цепочку отображений

$$
\mathscr{P}_{1} \stackrel{\mathscr{Q}_{2}}{\longrightarrow} \mathscr{P}_{2} \stackrel{\mathscr{Q}_{1}}{\longrightarrow} \mathscr{P}_{1} \stackrel{\mathscr{Q}_{1}}{\longrightarrow} \mathscr{P}_{2} \stackrel{\mathscr{Q}_{3}}{\longrightarrow} \mathscr{P}_{1} \stackrel{\mathscr{Q}_{3}}{\longrightarrow} \mathscr{P}_{2} \stackrel{\mathscr{Q}_{2}}{\longrightarrow} \mathscr{P}_{1},
$$

которая определяет левую часть формулы (4). Композиции второго и третьего, а также четвертого и пятого отображений в этой цепочке дают тождественное отображение. После их сокращения композиция первого и последнего отображения также дает тождественное отображение пространства $\mathscr{P}_{1}$ на себя.

Поясним геометрический смысл леммы 4. Рассмотрим каноническое (тавтологическое) расслоение $\gamma$ на многообразии $\mathrm{GrS}$, т.е. такое расслоение, для которого слоем над точкой $W \in \mathrm{GrS}$ служит линейное пространство $W$. Введем следующую локальную тривиализацию $\gamma$. Зафиксируем точку $W_{+} \in \mathrm{GrS}$. Карта $\mathscr{U}_{V}$ на $\gamma$ определяется заданием плоскости $V \in H$, являющейся дополнением к $W_{+}$, т.е. $W_{+} \oplus V=H$, причем нам будет удобно дополнительно потребовать, чтобы оператор проектирования $\pi_{V}: H \rightarrow W_{+}$параллельно пространству $V$ был ограниченным. Иными словами, для разложения $h=w+v$, где $w \in W_{+}, \quad v \in V$, имеет место оценка $\|w\| \leqslant C\|h\|$ с некоторой константой $C$ (подпространство $V$ не имеет “сколь угодно малых углов" с $W_{+}$). Координатами точки $(W, x) \in \gamma$, где $x \in W$, в карте $\mathscr{U}_{V}$ будут $\left(W, \pi_{V} x\right) \in \operatorname{GrS} \times W_{+}$. Вычислим функцию перехода от карты, определяемой плоскостью $V_{1}$, к карте, определяемой плоскостью $V_{2}$. Пусть точка $(W, x) \in \gamma$ имеет в карте $\mathscr{U}_{V_{1}}$ координаты $(W, y)$, где $y \in W_{+}$. Тогда $x=\pi_{V_{1}}^{-1} y$ и в карте $\mathscr{U}_{V_{2}}$ та же точка имеет координаты $\left(W, \pi_{V_{2}} \circ \pi_{V_{1}}^{-1} y\right)$. Но $\pi_{V}=\pi_{V}^{-1}$, поскольку $\pi_{V}$ - проектор. Следовательно, функция перехода задается отображением

$$
W_{+} \stackrel{V_{1}}{\longrightarrow} W \stackrel{V_{2}}{\longrightarrow} W_{+},
$$

которое совпадает с двойным отношением четверки подпространств $\mathrm{DV}\left(W_{+}\right.$, $\left.V_{2} ; W, V_{1}\right)$. По лемме 4 переход из карты $\mathscr{U}_{V_{1}}$ в карту $\mathscr{U}_{V_{2}}$, далее из $\mathscr{U}_{V_{2}}$ в $\mathscr{U}_{V_{3}}$ и, наконец, из $\mathscr{U}_{V_{3}}$ в $\mathscr{U}_{V_{1}}$ дает свойство коцикла (4) для функций перехода.

Мы показали, что при переходе от карты $\mathscr{U}_{V_{1}}$ к карте $\mathscr{U}_{V_{2}}$ на координату $x \in W$ действует преобразование $\mathrm{DV}\left(W_{+}, V_{2} ; W, V_{1}\right)$. Это преобразование определено на пересечениях карт типа $\mathscr{U}_{V}$. Но совокупность этих карт покрывает не все грассманово многообразие. В отличие от конечномерного случая в бесконечномерном гильбертовом пространстве два изоморфных подпространства, вообще говоря, могут не иметь общего для обоих дополнительного подпространства $V$. Точные необходимые и достаточные условия, которым должны удовлетворять два подпространства для того, чтобы они имели общее дополнение, было найдено в работе [9]. Чтобы обойти возникшую трудность, заменим двойное отношение $\operatorname{DV}\left(W_{+}, V_{2} ; W, V_{1}\right)$ на $\operatorname{DV}\left(W, V_{1} ; W_{+}, V_{2}\right)$. В силу леммы 1 двойное отношение сохраняется, но теперь оно определяет преобразование пространства $W$, а не $W_{+}$. Мы получаем возможность переходить от одного подпространства $W_{+}$к другому, и теперь эти карты покрывают все грассманово многообразие. 
Итак, переходы от одной карты к другой определяют эндоморфизмы $\operatorname{DV}\left(W, V_{1} ; W_{+}, V_{2}\right)$ пространства $W$, т.е. функции перехода канонического расслоения $\gamma$. В силу леммы 4 двойное отношение определяет на грассманиане коцикл $\{\mathrm{DV}\}$ с коэффициентами в пучке эндоморфизмов канонического расслоения $\gamma$, т.е.

$$
\{\mathrm{DV}\} \in H^{1}(\mathrm{GrS}, \operatorname{End}(\gamma)) .
$$

У нас появляется возможность дать гомологическую интерпретацию операторного двойного отношения.

Следуя схеме Атьи [10] и Тюрина [11], рассмотрим главное расслоение $P$ со структурной группой $G$ автоморфизмов слоя, соответствующее векторному расслоению $\gamma$. Образуем точную последовательность расслоений над базой грассманиана Сато GrS.

$$
0 \longrightarrow L \stackrel{f}{\longrightarrow} Q \stackrel{g}{\longrightarrow} T \longrightarrow 0
$$

где $T$ - касательное расслоение к $\mathrm{GrS} ; Q$ - расслоение $G$-инвариантных векторных полей, касательных к $P ; L$ - расслоение $G$-инвариантных вертикальных векторных полей (т.е. полей, касательных к слоям расслоения $P$ ). Последовательность (5) определяет расширение $T$ с помощью $L$. Классы эквивалентных расширений находятся во взаимно однозначном соответствии с элементами $H^{1}(\mathrm{GrS}, \operatorname{Hom}(T, L))$ - одномерной группы когомологий с коэффициентами в пучке $\operatorname{Hom}(T, L)$. Как и в конечномерном случае, назовем связностъю расщепляющий гомоморфизм $h: T \rightarrow Q$ такой, что $g \circ h=\mathrm{Id}$. Обозначим через $a(P) \in H^{1}(\mathrm{GrS}, \operatorname{Hom}(T, L))$ элемент, отвечающий расширению (5).

Рассмотрим простое покрытие базы расслоения $P$ (т.е. покрытие, состоящее из стягиваемых окрестностей). Функции перехода $\varphi_{i j}=u_{i}^{-1} u_{j}$ из карты $u_{i}: U_{i} \times\left. G \rightarrow P\right|_{U_{i}}$ в карту $u_{j}: U_{j} \times\left. G \rightarrow P\right|_{U_{j}}$ расслоения $P$ задаются двойным отношением $\varphi_{i j}=\mathrm{DV}\left(W, V_{i} ; W_{+}, V_{j}\right)$. Карта $u_{i}$ индуцирует изоморфизм касательных расслоений, и, поскольку он коммутирует с действием $G$, мы получаем изоморфизм $\widehat{u}_{i}: T_{i} \oplus L_{i} \rightarrow Q_{i}$, где через $T_{i}, Q_{i}, L_{i}$ обозначены ограничения соответствующих расслоений на окрестность $U_{i}$. На этой окрестности последовательность (5) расщепляется, поэтому существует подъем тождественного эндоморфизма расслоения $T$, дающий элемент $a_{i}: T_{i} \rightarrow Q_{i}$, а именно $a_{i}(t)=\widehat{u}_{i}(t \oplus 0)$. Положим $a_{i j}=\left(a_{j}-a_{i}\right): T_{i j} \rightarrow Q_{i j}$. Тогда $\left\{a_{i j}\right\}$ представляет коцикл $a(P)$.

Пусть $\Omega^{1}$ - пучок ростков дифференциальных 1-форм на многообразии $\mathrm{GrS}$. Имеем $\Omega^{1}=\operatorname{Hom}(T, \mathbf{1})$. Поэтому $H^{1}(\mathrm{GrS}, \operatorname{Hom}(T, L))=H^{1}\left(\mathrm{GrS}, L \otimes \Omega^{1}\right)$. Итак, $a(P) \in H^{1}\left(\mathrm{GrS}, L \otimes \Omega^{1}\right)$. Для компактных кэлеровых многообразий в силу изоморфизма Дольбо $H^{1}\left(\mathrm{GrS}, L \otimes \Omega^{1}\right)$ соответствует когомологиям типа $H^{1,1}$ и порождает кольцо характеристических классов расслоения. В нашем случае (многообразие $\mathrm{GrS}$ не компактно) будем по определению считать $a(P)$ аналогом порождающего класса Чженя для канонического расслоения $\gamma$.

\section{§ 3. Двойное отношение как инвариант унитарной ограниченной линейной группы}

Группа $U_{\text {res }}$ действует на большой клетке грассманиана Сато транзитивно, но не дважды транзитивно, как известно уже для конечномерного случая. Естественно поставить вопрос об инвариантах пары точек относительно действия группы $U_{\text {res. }}$. Эти инварианты строятся аналогично конечномерному случаю [4] и тесно связаны с операторным двойным отношением. 
Пусть $\mathscr{A}, \mathscr{B} \in \mathrm{Gr}_{+}(H)$. Их координатами служат операторы ГильбертаШмидта $A$ и $B$ соответственно. Верхним индексом * будем обозначать сопряженный оператор.

ОПРЕДЕЛЕНИЕ 3. Оператором углов между подпространствами $\mathscr{A}$ и $\mathscr{B}$ назовем оператор

$$
\mathrm{DV}_{A B}:=\left(\operatorname{Id}+A^{*} A\right)^{-1}\left(\operatorname{Id}+A^{*} B\right)\left(\operatorname{Id}+B^{*} B\right)^{-1}\left(\operatorname{Id}+B^{*} A\right) .
$$

Отметим, что оператор $\left(\mathrm{Id}+A^{*} A\right)$ положительно определенный и обратимый. В конечномерном случае $\mathrm{DV}_{A B}$ задает квадраты косинусов углов векторов $a \in A$ с подпространством $B$ (см. [4]).

ОПредЕЛЕНиЕ 4. Две пары подпространств $\mathscr{S}, \mathscr{T}$ и $\mathscr{P}, \mathscr{Q}$ назовем эквивалентными относительно $U_{\text {res }}$, если существует такой элемент $g \in U_{\text {res }}$, что $g \mathscr{S}=\mathscr{P}, g \mathscr{T}=Q$.

ОПРеДЕЛЕНИЕ 5. Два оператора $V, W: H_{+} \rightarrow H_{-}$назовем сопоставимыми, если существуют такие унитарные операторы $\alpha \in U\left(H_{-}\right)$и $\beta \in U\left(H_{+}\right)$, что $W=\alpha V \beta$.

Теорема 2. Две парь точек $\mathscr{P}, \mathscr{Q}$ и $\mathscr{S}, \mathscr{T}$ грассманиана Сато эквивалентны тогда и только тогда, когда операторы $\mathrm{DV}_{P Q}$ u $\mathrm{DV}_{S T}$ совпадают.

Докажем сначала следующую лемму.

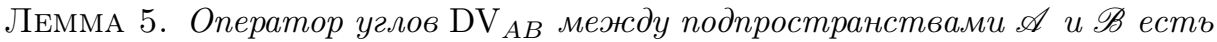
оператор сквозного отображения

$$
\mathscr{A} \stackrel{\mathscr{B}}{\longrightarrow} \mathscr{B} \stackrel{\mathscr{A}^{\perp}}{\longrightarrow} \mathscr{A} .
$$

Доказательство. Разложим элемент $(h, A h)$ подпространства $\mathscr{A}$ по базису $\mathscr{B}, \mathscr{B} \perp$. Если $(x, B x)$ есть проекция на $\mathscr{B}$, то векторы вида $((h, A h)-(x, B x))$ ортогональны $\mathscr{B}$, т.е. $\langle(h-x, A h-B x),(y, B y)\rangle=0$ для любого $y$, где угловыми скобками обозначено скалярное произведение в $H$. Отсюда получаем $x=\left(\operatorname{Id}+B^{*} B\right)^{-1}\left(\mathrm{Id}+B^{*} A\right) h$. Разлагая полученный элемент по базису $\mathscr{A}, \mathscr{A}^{\perp}$, получаем, аналогично, элемент $(z, A z)$, где

$$
z=\left(\operatorname{Id}+A^{*} A\right)^{-1}\left(\operatorname{Id}+A^{*} B\right)\left(\operatorname{Id}+B^{*} B\right)^{-1}\left(\operatorname{Id}+B^{*} A\right) h .
$$

СЛЕДСТВИЕ 1. Подпространства $\mathscr{A}^{\perp}, \mathscr{B}^{\perp}$ принадлежат грассманиану, двойственному $к$ грассманиану Сато, и оператор углов есть двойное отношение четверки подпространств $\mathscr{A}, \mathscr{A}^{\perp}, \mathscr{B}, \mathscr{B}^{\perp}$.

ДоКАЗАТЕЛЬСТво теОРЕМЫ 2. Необходимость следует из леммы 5 в силу инвариантности соответствующего сквозного отображения.

Достаточность. Поскольку $U_{\text {res }}$ действует на большой клетке транзитивно, можно считать, что $\mathscr{S}=\mathscr{P}$. Примем координаты этого подпространства в грассманиане Сато за нуль. Обозначим координаты подпространства $\mathscr{T}$ в этой карте через $W$, а координаты $\mathscr{Q}$ - через $V$. Тогда $\operatorname{DV}_{P Q}=\left(\operatorname{Id}+V^{*} V\right)^{-1}$, $\mathrm{DV}_{S T}=\left(\mathrm{Id}+W^{*} W\right)^{-1}$. Поскольку эти операторы подобны, то подобны и операторы $V^{*} V$ и $W^{*} W$. Эти операторы соответствуют квадратичным формам $\langle V x, V x\rangle$ и $\langle W x, W x\rangle$ на пространстве $H_{+}$.

Для продолжения доказательства нам понадобится следующая 
Лемма 6. Для того чтобъ две квадратичные формъ $\langle V x, V x\rangle u\langle W x, W x\rangle$ на пространстве $H_{+}$с операторами Гильберта-Шмидта $V, W: H_{+} \rightarrow H_{-}$ были подобны, необходимо и достаточно, чтобы операторы $V$ и $W$ были сопоставимы. Тогда

ДоказАтельство. Достаточность. Пусть $W=\alpha V \beta, \alpha^{*} \alpha=\mathrm{Id}, \beta^{*} \beta=\mathrm{Id}$.

$$
\left(W^{*} W\right)=\beta^{*} V^{*} \alpha^{*} \alpha V \beta=\beta^{*}\left(V^{*} V\right) \beta .
$$

Необходимость. Пусть $\left(W^{*} W\right)=\beta^{*}\left(V^{*} V\right) \beta$. Тогда $\operatorname{ker} W=\beta(\operatorname{ker} V)$. Умножая оператор $V$ на унитарный оператор $\beta$ справа, добиваемся совпадения ядер: $\operatorname{ker} W=\operatorname{ker} V_{1}$, где $V_{1}=V \beta$. В силу уже доказанной достаточности операторы $V_{1}^{*} V_{1}$ и $W^{*} W$ подобны. Умножим слева оператор $V_{1}$ на унитарный оператор $\gamma: H_{-} \rightarrow H_{-}$, который переводит образ $\operatorname{Im} V$ в $\operatorname{Im} W$. Обозначим $\gamma V_{1}=V_{2}$. Операторы $V_{2}^{*} V_{2}$ и $W^{*} W$ по-прежнему остаются подобными. Оператор $\widetilde{V}:(\operatorname{ker} W)^{\perp} \rightarrow \operatorname{Im} W$, являющийся ограничением оператора $V_{2}$, обратим. Через $\widetilde{W}$ обозначим ограничение оператора $W$ на подпространство $(\operatorname{ker} W)^{\perp}$. Операторы $\widetilde{V}$ и $\widetilde{W}$ эквивалентны. Следовательно, существует такое унитарное преобразование $q$ пространства $(\operatorname{ker} W)^{\perp}$, что

$$
\widetilde{W} \widetilde{W}^{*}=q^{*} \widetilde{V} q
$$

Возьмем $\alpha_{1}=q^{*}, \alpha_{2}=\widetilde{W} q^{*} \widetilde{V}^{-1}$. Тогда, во-первых, $\alpha_{2} \widetilde{V} \alpha_{1}=\widetilde{W}$, т.е. $\widetilde{V}$ и $\widetilde{W}$ сопоставимы, и, во-вторых, $\alpha_{2}^{*} \alpha_{2}=\left(\widetilde{V}^{*}\right)^{-1} q \widetilde{W}^{*} \widetilde{W} q^{*} \widetilde{V}^{-1}=\operatorname{Id}$ в силу (6), т.е. оператор $\alpha_{2}$ унитарный. Доопределив $\alpha_{1}$ и $\alpha_{2}$ на ядре единичными операторами, получаем, что $V$ и $W$ сопоставимы.

Для завершения доказательства теоремы остается заметить, что стабилизатор точки 0 в $U_{\text {res }}$ - это блочно-диагональные операторы

$$
\left(\begin{array}{cc}
\alpha_{1} & 0 \\
0 & \alpha_{2}
\end{array}\right),
$$

которые переводят координаты $V$ подпространства $\mathscr{Q}$ в сопоставимый оператор, следовательно, пара подпространств $\mathscr{P}, \mathscr{Q}$ переходит в пару $\mathscr{S}, \mathscr{T}$, что и требовалось доказать.

УтвеРЖДЕНИЕ 1. Группа $\mathrm{GL}_{\mathrm{res}}(H)$ транзитивно действует на грассманиане $\widetilde{\mathrm{GrS}}$, двойственном $\kappa$ грассманиану Сато.

ДоказАтельство. Пусть $\mathscr{P} \in \mathrm{GrS}$. Тогда по определению ортогональная проекция $\mathrm{pr}_{-}: \mathscr{P} \rightarrow W_{+}$есть оператор Фредгольма; а ортогональная проекция $\mathrm{pr}_{+}: \mathscr{P} \rightarrow W_{-}$есть оператор Гильберта-Шмидта. Поскольку $W_{-}=W_{+}^{\perp}$, имеем $\operatorname{DV}_{\mathscr{P} W_{+}}=\operatorname{DV}\left(\mathscr{P}, \mathscr{P}^{\perp} ; W_{+}, W_{-}\right)$. Используя леммы 1-3, преобразуем правую часть:

$$
\operatorname{DV}\left(\mathscr{P}, \mathscr{P}^{\perp} ; W_{+}, W_{-}\right)=\mathrm{DV}\left(\mathscr{P}^{\perp}, \mathscr{P} ; W_{-}, W_{+}\right)=\mathrm{DV}_{\mathscr{P}}{ }^{\perp} W_{-} .
$$

Следовательно, в силу теоремы 2 пары подпространств $\mathscr{P}, W_{+}$и $\mathscr{P} \perp, W_{-}$, имея одинаковый оператор углов, эквивалентны. Следовательно, существует оператор $\mathfrak{A} \in U_{\text {res }}$, переводящий одну из этих пар в другую. Значит, из того, что проекция $\mathrm{pr}_{-}: \mathscr{P} \rightarrow W_{+}$фредгольмова, следует, что проекция $\mathrm{pr}_{+}: \mathscr{P} \perp \rightarrow W_{-}$ 
есть также оператор Фредгольма. Точно так же доказывается эквивалентность пар подпространств $\mathscr{P}, W_{-}$и $\mathscr{P} \perp, W_{+}$, из которой следует, что проекция $\mathrm{pr}_{-}: \mathscr{P}^{\perp} \rightarrow W_{+}$есть оператор Гильберта-Шмидта. Следовательно, подпространство $\mathscr{P} \perp$ принадлежит грассманиану Сато, построенному по поляризации с измененной ролью подпространств $W_{+}$и $W_{-}$, откуда и следует доказываемое утверждение.

В случае, когда подпространства, принадлежащие двойственному грассманиану $\widetilde{\operatorname{Gr}}(H)$, не изоморфны подпространствам из $\operatorname{Gr}(H)$ (например, имеют разную размерность), формула (2) теряет смысл. Выведем формулу, дающую координатное представление оператора DV в общем случае. Пусть $H=H_{+} \oplus H_{-}-$поляризация пространства $H$. Будем считать, что подпространства $\mathscr{P}_{+}$и $\mathscr{Q}_{+}$сравнимы с $H_{+}$, а $\mathscr{P}_{-}$и $\mathscr{Q}_{-}$сравнимы с $H_{-}$. Это означает, что $\mathscr{P}_{+}, \mathscr{Q}_{+}$- точки грассманиана $\operatorname{Gr}(H)$, а $\mathscr{P}_{-}, \mathscr{Q}_{-}-$точки двойственного грассманиана $\widetilde{\mathrm{Gr}}(H)$. Пусть координатами подпространств $\mathscr{P}_{+}, \mathscr{Q}_{+}$служат отображения $P_{+}: H_{+} \rightarrow H_{-}$и $Q_{+}: H_{+} \rightarrow H_{-}$соответственно. Для подпространств $\mathscr{P}_{-}$и $\mathscr{Q}_{-}$переменим роли вертикального и горизонтального подпространств $H_{+}$и $H_{-}$, и пусть в этой системе координат подпространства $\mathscr{P}_{-}$и $\mathscr{Q}_{-}$имеют своими координатами отображения $P_{-}: H_{-} \rightarrow H_{+}$и $Q_{-}: H_{-} \rightarrow H_{+}$соответственно.

Заметим, что произведение отображений $P_{+} P_{-}$задает отображение подпространства $H_{-}$в себя, а произведение $P_{-} P_{+}$- отображение подпространства $H_{+}$ в себя. В частности, в конечномерном случае если $P_{+}-$прямоугольная $(n \times m)$ матрица, то $P_{+} P_{-}-$квадратная $(n \times n)$-матрица.

Лемма 7. Подпространства $\mathscr{P}_{+}$и $\mathscr{P}_{-}$имеют непустое пересечение тогда и только тогда, когда композичия операторов $P_{-} P_{+}$(эквивалентно, $\left.P_{+} P_{-}\right)$ имеет единичу в качестве своего собственного значения.

ДоказАтельство. Подпространства $\mathscr{P}_{+}$и $\mathscr{P}_{-}$имеют непустое пересечение, если и только если существуют $x \in H_{+}$и $y \in H_{-}$, для которых $\left(x, P_{+} x\right)=$ $\left(P_{-} y, y\right)$. Это означает, что $x=P_{-} y, P_{+} x=y$, что в свою очередь эквивалентно соотношениям $x=P_{-} P_{+} x, P_{+} P_{-} y=y$.

УтВеРЖДЕНИЕ 2. Пусть $\mathscr{P}_{+}, \mathscr{P}_{-}$u $\mathscr{Q}_{+}, \mathscr{Q}_{-}-$nоляризаиии, и пусть проекции параллельно $\mathscr{P}_{-}$и $\mathscr{Q}_{-}-$изоморфизмы пространств $\mathscr{P}_{+} u \mathscr{Q}_{+}$.

тогда $\operatorname{DV}\left(\mathscr{P}_{+}, \mathscr{P}_{-} ; \mathscr{Q}_{+}, \mathscr{Q}_{-}\right)$- сквозное отображсение $\mathscr{P}_{+} \stackrel{\mathscr{Q}_{-}}{\longrightarrow} \mathscr{Q}_{+} \stackrel{\mathscr{P}_{-}}{\longrightarrow} \mathscr{P}_{+}$ пространства $\mathscr{P}_{+}$на себя-имеет вид

$$
\left(P_{-} P_{+}-I\right)^{-1}\left(P_{-} Q_{+}-I\right)\left(Q_{-} Q_{+}-I\right)^{-1}\left(Q_{-} P_{+}-I\right) .
$$

ДоказАтельство. Пусть $\left(g, Q_{+} g\right)$ - проекция элемента $\left(f, P_{+} f\right)$ пространства $\mathscr{P}_{+}$на $\mathscr{Q}_{+}$параллельно $\mathscr{Q}_{-}$. Это означает, что $\left(f-g, P_{+} f-Q_{+} g\right) \in \mathscr{Q}_{-}$. Поскольку для $\mathscr{Q}_{-}$горизонтальное и вертикальное подпространства поменялись ролями, имеем $Q_{-}\left(P_{+} f-Q_{+} g\right)=(f-g)$. Из леммы 7 следует, что оператор $\left(Q_{-} Q_{+}-I\right)$ обратим (проекция однозначно определена для любого вектора), и мы получаем $g=\left(Q_{-} Q_{+}-I\right)^{-1}\left(Q_{-} P_{+}-I\right) f$. Аналогично, проекция элемента $\left(g, Q_{+} g\right)$ на $\mathscr{P}_{+}$параллельно $\mathscr{P}_{-}$есть $\left(h, P_{+} h\right)$, где $h=$ $\left(P_{-} P_{+}-I\right)^{-1}\left(P_{-} Q_{+}-I\right) g=\left(P_{-} P_{+}-I\right)^{-1}\left(P_{-} Q_{+}-I\right)\left(Q_{-} Q_{+}-I\right)^{-1}\left(Q_{-} P_{+}-I\right) f$.

В теории вполне интегрируемых бесконечномерных гамильтоновых систем и в ряде квантово-механических моделей важную роль играет $\tau$-функция, зависящая от подпространств грассманиана Сато. По мнению ряда авторов 
$\tau$-функция должна играть в квантовой теории поля не меньшую роль, чем $\vartheta$-функция в алгебраической геометрии. Кстати сказать, в некоторых случаях $\tau$-функция совпадает с $\vartheta$-функцией. Используя результаты настоящей статьи и работу [8], нетрудно показать, что $\tau$-функция совпадает с детерминантом операторного двойного отношения двух пар точек $\mathrm{GrS}$ и $\widetilde{\mathrm{GrS}}$. Нам представляется, что использование операторного двойного отношения (а не только его детерминанта) должно давать существенно большую информацию.

Не нарушая сложившуюся лингвистическую традицию, будем называть операторное двойное отношение для грассманиана Сато "Тэ-функцией" и обозначать ее заглавной готической буквой $\mathfrak{T}$. В обозначениях утверждения 2 при $\mathscr{P}_{+}, \mathscr{Q}_{+} \in \mathrm{GrS}$ и $\mathscr{P}_{-}, \mathscr{Q}_{-} \in \widetilde{\mathrm{GrS}}$ имеем следующее определение:

$$
\mathfrak{T}\left(\mathscr{P}_{+}, \mathscr{P}_{-} ; \mathscr{Q}_{+}, \mathscr{Q}_{-}\right)=\left(P_{-} P_{+}-I\right)^{-1}\left(P_{-} Q_{+}-I\right)\left(Q_{-} Q_{+}-I\right)^{-1}\left(Q_{-} P_{+}-I\right) .
$$

При этом, как и в случае $\tau$-функции, одно из подпространств можно начать менять, используя группу, действующую на грассманиане Сато.

Как уже отмечалось,

$$
\tau\left(\mathscr{P}_{+}, \mathscr{P}_{-} ; \mathscr{Q}_{+}, \mathscr{Q}_{-}\right)=\operatorname{det} \mathfrak{T}\left(\mathscr{P}_{+}, \mathscr{P}_{-} ; \mathscr{Q}_{+}, \mathscr{Q}_{-}\right) .
$$

В физических приложениях $\tau$-функция играет существенную роль. В частности, в моделях квантовой теории поля [2], [12], точнее, для матричных моделей Ферми-жидкости - свободной системы фермионов в размерности $(1+1)-$ $\tau$-функция соответствует главному члену асимптотики квазиклассического приближения (при $\hbar \rightarrow 0$ ) вакуумного среднего значения внутренней энергии. А именно это вакуумное среднее выражается как $\exp \left(\hbar^{-2} \tau(x, t)+O\left(\hbar^{-1}\right)\right)$.

ГипотезА 1. T-функция задает главный член асимптотики оператора внутренней энергии при $\hbar \rightarrow 0$.

Выше было показано, что существенным объектом для исследования двойного отношения является многообразие $R$ поляризаций гильбертова пространства $H$ :

$$
R \subset \operatorname{Gr}(H) \times \widetilde{\operatorname{Gr}}(H),
$$

где $\widetilde{\operatorname{Gr}}(H)$ - грассманиан, двойственный к $\operatorname{Gr}(H)$. Тавтологическим расслоением $\rho$ над $R$ назовем расслоение, которое каждой точке $\mathscr{P}=\left(\mathscr{P}_{+}, \mathscr{P}_{-}\right) \in R$ ставит в соответствие слой $\mathscr{P}_{+}$. Рассмотрим главное расслоение $\mathscr{K}$, ассоциированное с $\rho$. Иными словами, слоем $\mathscr{K}$ над точкой $\mathscr{P}=\left(\mathscr{P}_{+}, \mathscr{P}_{-}\right) \in R$ служит группа $\operatorname{GL}\left(\mathscr{P}_{+}\right)$преобразований слоя $\mathscr{P}_{+} \cdot$ Двойное отношение порождает естественную связность на расслоении $\mathscr{K}$, что даст нам возможность вычислить кривизну этого расслоения.

Определим параллельный перенос на $\mathscr{K}$ с помощью проектирования на "близкое" к $\mathscr{P}_{+}$подпространство $\mathscr{Q}_{+}$параллельно некоторому подпространству $\mathscr{Q}_{-} \in \widetilde{\mathrm{Gr}}(H)$.

Фиксируем базисную поляризацию $\mathscr{Q}=\left(\mathscr{Q}_{+}, \mathscr{Q}_{-}\right) \in R$. Рассмотрим два локальных сечения $\alpha$ и $\beta$ расслоения $\mathscr{K}$. Пусть отображение $\alpha(\mathscr{P})$ - прообраз некоторого фиксированного преобразования слоя $\mathscr{Q}_{+}$расслоения $\rho$ над точкой $\mathscr{Q}$ при отображении $\mathscr{P}_{+} \stackrel{\mathscr{Q}_{-}}{\longrightarrow} \mathscr{Q}_{+}$. Отображение $\beta$ - аналогичный прообраз при отображении $\mathscr{P}_{+} \stackrel{\mathscr{P}_{-}}{\longrightarrow} \mathscr{Q}_{+}$. Отметим, что $\mathfrak{T}=\beta^{-1} \alpha$ и, следовательно,

$$
\alpha=\beta \mathfrak{T}, \quad \beta=\alpha \mathfrak{T}^{-1} .
$$


Обозначим ковариантную производную по направлениям $\mathscr{P}_{+}$через $\nabla_{+}$, ковариантную производную по направлениям $\mathscr{P}_{-}-$через $\nabla_{-}$.

Сечение $\alpha$ ковариантно постоянно вдоль сомножителя базы $\left\{\mathscr{P}_{+}\right\} \times \widetilde{\operatorname{Gr}}(H)$ с фиксированным $\mathscr{P}_{+}$; сечение $\beta$ ковариантно постоянно вдоль сомножителя базы $\operatorname{Gr}(H) \times\left\{\mathscr{P}_{-}\right\}$с фиксированным $\mathscr{P}_{-}$. Это означает, что $\nabla_{+} \beta=0$, $\nabla_{-} \alpha=0$. Вычислим ковариантную производную обеих частей первого равенства в формулах (8) по направлениям $\mathscr{P}_{+}$:

$$
\nabla_{+} \alpha=\beta \nabla_{+} \mathfrak{T}=\alpha \mathfrak{T}^{-1} \nabla_{+} \mathfrak{T} .
$$

Коэффициент при $\alpha$ в правой части формулы (9) соответствует связности расслоения $\mathscr{K}$. Обозначим его через $\omega_{+}=\mathfrak{T}^{-1} \nabla_{+} \mathfrak{T}$. Дифференцируя (7), находим

$$
\begin{aligned}
\nabla_{+} \mathfrak{T}=- & \left(P_{-} P_{+}-1\right)^{-1} P_{-} d P_{+}\left(P_{-} P_{+}-1\right)^{-1} \\
& \times\left(P_{-} Q_{+}-1\right)\left(Q_{-} Q_{+}-1\right)^{-1}\left(Q_{-} P_{+}-1\right) \\
& +\left(P_{-} P_{+}-1\right)^{-1}\left(P_{-} Q_{+}-1\right)\left(Q_{-} Q_{+}-1\right)^{-1} Q_{-} d P_{+} .
\end{aligned}
$$

Следовательно, значение формы связности на векторах из $\mathscr{P}_{+}$равно

$$
\begin{aligned}
\omega_{+}=- & \left(P_{-} P_{+}-1\right)^{-1} P_{-} d P_{+}+\left(P_{-} P_{+}-1\right)^{-1}\left(P_{-} Q_{+}-1\right)\left(Q_{-} Q_{+}-1\right)^{-1} Q_{-} d P_{+} \\
& +\left(Q_{-} P_{+}-1\right)^{-1}\left(Q_{-} Q_{+}-1\right)\left(P_{-} Q_{+}-1\right)^{-1}\left(P_{-} P_{+}-1\right) .
\end{aligned}
$$

Для того чтобы вычислить кривизну $\Omega$ связности $\omega$, надо взять от нее внешнюю ковариантную производную $d=\partial_{+}+\partial_{-}$, где через $\partial_{+}$обозначена ковариантная производная по направлениям $\mathscr{P}_{+}$, а через $\partial_{-}-$ковариантная производная по направлениям $\mathscr{P}_{-}$. Вычисление соответствующих производных от $\omega_{+}$с последующей подстановкой значений $P_{+}=Q_{+}, P_{-}=Q_{-}$дает

$$
\begin{aligned}
\partial_{+} \omega_{+}= & 0 \\
\partial_{-} \omega_{+}= & \left(Q_{-} Q_{+}-1\right)^{-1} d Q_{-} Q_{+}\left(Q_{-} Q_{+}-1\right)^{-1} Q_{-} d Q_{+} \\
& \quad-\left(Q_{-} Q_{+}-1\right)^{-1} d Q_{-} d Q_{+} .
\end{aligned}
$$

Итак, форма кривизны для расслоения $\mathscr{K}$ равна

$$
\Omega=\left(Q_{-} Q_{+}-1\right)^{-1} d Q_{-} Q_{+}\left(Q_{-} Q_{+}-1\right)^{-1} Q_{-} d Q_{+}-\left(Q_{-} Q_{+}-1\right)^{-1} d Q_{-} d Q_{+} .
$$

Формула (10) является обобщением не только на конечномерную, но и на бесконечномерную ситуацию широко известной для одномерной $\tau$-функции формулы $\Omega=\partial_{+} \partial_{-} \log \tau$ (см. [8], наше вычисление обобщает вычисления, проделанные в этой работе).

В точности то же значение кривизны можно получить, вычисляя ковариантную производную второго равенства в формулах (8) по направлениям $\mathscr{P}_{-}$.

$\mathrm{K}$ вышеописанной конструкции тесно примыкает понятие производной Шварца, операторное обобщение которой мы определим в следующем параграфе.

\section{§ 4. Оператор Шварца}

Операторное двойное отношение позволяет определить операторный аналог дифференциального оператора Шварца для кривых на грассмановом многообразии (см. [4] и цитируемую там литературу). 
Пусть $H$ - гильбертово пространство, оснащенное поляризацией $H=$ $H_{+} \oplus H_{-}$. Рассмотрим грассманово многообразие $\operatorname{Gr}(H)$, определяемое подпространством $H_{+}$, и двойственное к нему грассманово многообразие $\widetilde{\operatorname{Gr}}(H)$, состоящее из подпространств, дополняющих элементы $\operatorname{Gr}(H)$ до полного пространства $H$. Будем считать, что подпространства, принадлежащие $\operatorname{Gr}(H)$, изоморфны подпространствам, принадлежащим $\widetilde{\mathrm{Gr}}(H)$. В конечномерном случае это означает, что размерности $H_{+}$и $H_{-}$одинаковы.

Рассмотрим в пространстве $H$ гладкое семейство подпространств $\mathscr{Z}(s)$, зависящих от одномерного параметра $s$, такое, что пары $(\mathscr{Z}(s), \mathscr{Z}(\sigma))$ при $s>0$ и $\sigma \leqslant 0$ образуют поляризации пространства $H$. Наша ближайшая цель построить аналог производной Шварца кривой $\mathscr{Z}(s)$ при $s=0$.

Пусть $s_{2}<0<s_{1}<s_{3}$. Тогда пары подпространств $\left(\mathscr{Z}\left(s_{2}\right), \mathscr{Z}\left(s_{1}\right)\right)$ и $\left(\mathscr{Z}(0), \mathscr{Z}\left(s_{3}\right)\right)$ задают поляризации $H$. В дальнейшем вместо $\mathscr{Z}\left(s_{i}\right), \mathscr{Z}^{\prime}\left(s_{i}\right), \ldots$ (штрих соответствует дифференцированию по $s$ ) будем для краткости писать $\mathscr{Z}_{i}, \mathscr{Z}_{i}^{\prime}, \ldots$; значения $\mathscr{Z}(0), \mathscr{Z}^{\prime}(0), \ldots$ будем обозначать просто через $\mathscr{Z}, \mathscr{Z}^{\prime}, \ldots$ (без индекса). Координаты этих пространств будем обозначать соответствующими строчными буквами $z, z_{i}, z_{i}^{\prime}, \ldots$. Рассмотрим отображение $f$, зависящее от четырех параметров и задаваемое двойным отношением

$$
f\left(s_{2}, s_{1}, 0, s_{3}\right)=\operatorname{DV}\left(\mathscr{Z}_{2}, \mathscr{Z}_{1} ; \mathscr{Z}, \mathscr{Z}_{3}\right)=\left(z_{2}-z_{1}\right)^{-1}\left(z_{1}-z\right)\left(z-z_{3}\right)^{-1}\left(z_{3}-z_{2}\right) .
$$

Устремим сначала $s_{2}$ к нулю. При $s_{2}=0$ двойное отношение $f$ задает тождественное отображение. Производная $f$ в нуле равна

$$
\frac{\partial f}{\partial s_{2}}\left(0, s_{1} ; 0, s_{3}\right)=-\left(z-z_{1}\right)^{-1} z^{\prime}+\left(z-z_{3}\right)^{-1} z^{\prime} .
$$

Устремим теперь $s_{3}$ к $s_{1}$. При $s_{3}=s_{1}$ функция $f$ и ее производная по $s_{2}$ равны нулю. Имеем

$$
\frac{\partial^{2} f}{\partial s_{3} \partial s_{2}}\left(0, s_{1} ; 0, s_{1}\right)=-\left(z-z_{1}\right)^{-1} z_{1}^{\prime}\left(z-z_{1}\right)^{-1} z^{\prime} .
$$

Правая часть формулы (11) определена при $s_{1}>0$ и имеет особенность при $s_{1}=0$. Рассмотрим процесс "схлопывания" поляризаций: найдем асимптотику (11) при $s_{1} \rightarrow 0$. Имеем

$$
\begin{aligned}
z_{1}^{\prime} & =z^{\prime}+s_{1} z^{\prime \prime}+\frac{s_{1}^{2} z^{\prime \prime \prime}}{2}+o\left(s_{1}^{2}\right), \\
z_{1}-z & =s_{1} z^{\prime}\left(\operatorname{Id}+\frac{s_{1}\left(z^{\prime}\right)^{-1} z^{\prime \prime}}{2}+\frac{s_{1}^{2}\left(z^{\prime}\right)^{-1} z^{\prime \prime \prime}}{6}+o\left(s_{1}^{2}\right)\right), \\
\left(z_{1}-z\right)^{-1} & =s_{1}^{-1}\left(\operatorname{Id}-\frac{s_{1}\left(z^{\prime}\right)^{-1} z^{\prime \prime}}{2}-\frac{s_{1}^{2}\left(z^{\prime}\right)^{-1} z^{\prime \prime \prime}}{6}+\frac{s_{1}^{2}\left(\left(z^{\prime}\right)^{-1} z^{\prime \prime}\right)^{2}}{4}+o\left(s_{1}^{2}\right)\right)\left(z^{\prime}\right)^{-1} .
\end{aligned}
$$

Подставив эти выражения в (11), после перемножения получаем

$$
\frac{\partial^{2} f}{\partial s_{3} \partial s_{2}}(0,0 ; 0,0)=\left(s_{1}\right)^{-2}\left(\operatorname{Id}+\frac{s_{1}^{2}\left(z^{\prime}\right)^{-1} z^{\prime \prime \prime}}{6}-\frac{s_{1}^{2}\left(\left(z^{\prime}\right)^{-1} z^{\prime \prime}\right)^{2}}{4}+o\left(s_{1}^{2}\right)\right) \text {. }
$$

В соответствии с этой формулой определим оператор дифференцирования

$$
S(z)=\left(z^{\prime}\right)^{-1} z^{\prime \prime \prime}-\frac{3}{2}\left(\left(z^{\prime}\right)^{-1} z^{\prime \prime}\right)^{2} .
$$


Получилось выражение, аналогичное производной Шварца. В конечномерном случае оно было введено и изучалось нами в работе [4]. Приведенный вывод показывает, что производная Шварца определена и в бесконечномерном случае, причем выражение (12) так же, как и двойное отношение, с помощью которого оно построено, однозначно определяет один и тот же класс сопряженных операторов независимо от преобразований Мёбиуса в объемлющем пространстве.

Покажем, что в конечномерном случае производная (12) тесно связана с гамильтоновыми системами и с уравнением Риккати.

Пусть

$$
\left\{\begin{array}{l}
q^{\prime}=A(t) q+p \\
p^{\prime}=-B(t) q-A^{*}(t) p
\end{array}\right.
$$

- линейная гамильтонова система с гамильтонианом

$$
H=\frac{1}{2}(\langle p, p\rangle+2\langle p, A q\rangle+\langle B q, q\rangle),
$$

где $A(t)$ и $B(t)$ являются $(n \times n)$-матрицами, матрица $B(t)$ симметрическая, угловые скобки - стандартное скалярное произведение в $\mathbb{R}^{n}$. Гамильтониан (14) соответствует задаче минимизации квадратичного функционала

$$
\int_{t_{0}}^{t_{1}}\left(\left\langle q^{\prime}, q^{\prime}\right\rangle-2\left\langle A q, q^{\prime}\right\rangle+\left\langle\left(A^{*} A-B\right) q, q\right\rangle\right) d t .
$$

Единичная матрица у первого слагаемого подынтегрального выражения в формуле (15) - результат редукции вариационной задачи, удовлетворяющей усиленному условию Лежандра. Система (13) эквивалентна уравнению Эйлера для функционала (15):

$$
q^{\prime \prime}+\left(A^{*}-A\right) q^{\prime}+\left(B-A^{\prime}-A^{*} A\right) q=0 .
$$

Мы будем рассматривать фундаментальные системы решений уравнений (13), поэтому $p$ и $q$ будут тоже $(n \times n)$-матрицами.

Матрица коэффициентов системы (13) является симплектической, поэтому (13) определяет поток на многообразии лагранжевых плоскостей пространства $(p, q)$, т.е. на многообразии Лагранжа-Грассмана $\Lambda$. Точки многообразия $\Lambda$ в локальных координатах задаются матрицами $W=p q^{-1}$. Эволюция координат $W$ описывается уравнением Риккати

$$
W^{\prime}=\left(-B q-A^{*} p\right) q^{-1}-p q^{-1}(A q+p) q^{-1}=-B-A^{*} W-W A-W^{2} .
$$

Вернемся к матричному аналогу дифференциального оператора Шварца, который переводит матричнозначную функцию $z(t)$ в функцию

$$
S(z(t))=\left[\left(z^{\prime}(t)\right)^{-1} z^{\prime \prime}\right]^{\prime}-\frac{1}{2}\left[\left(z^{\prime}(t)\right)^{-1} z^{\prime \prime}\right]^{2}=\left(z^{\prime}(t)\right)^{-1} z^{\prime \prime \prime}-\frac{3}{2}\left[\left(z^{\prime}(t)\right)^{-1} z^{\prime \prime}\right]^{2} .
$$

Удобно считать, что переменная $t$ меняется на проективной прямой (действительной или комплексной) или на римановой поверхности, на которой действует группа Мёбиуса дробно-линейных преобразований.

В [4] было доказано, что класс эквивалентности образа $S(z(\cdot))$ любой конкретной функции $z(\cdot)$ инвариантен относительно группы Мёбиуса, т.е. 
если обозначить через $M$ преобразование Мёбиуса $M: z \mapsto\left(C_{1} z+C_{2}\right) \times$ $\left(C_{3} z+C_{4}\right)^{-1}$, где $C_{i}$ - это $(n \times n)$-матрицы, то существует такая матрица $K(t)$, что $S(M(z(t)))=K(t) S(z(t)) K^{-1}(t)$. Иными словами, преобразования Мёбиуса прообраза оператора Шварца приводят лишь к изоспектральному изменению его образа.

Опишем связь оператора $S$ с гамильтоновой системой (13) или, что то же, с уравнением Риккати (17). Предположим, что матрица $A$ симметрична. Зададим связь между $W$, являющейся решением уравнения $(17)$, и функцией $z$ формулой

$$
W=-\frac{1}{2}\left[\left(z^{\prime}(t)\right)^{-1} z^{\prime \prime}\right]-A .
$$

Имеем

$$
\begin{aligned}
W^{\prime} & =-\frac{1}{2}\left(z^{\prime}(t)\right)^{-1} z^{\prime \prime \prime}+\frac{1}{2}\left(z^{\prime}(t)\right)^{-1} z^{\prime \prime}\left(z^{\prime}(t)\right)^{-1} z^{\prime \prime}-A^{\prime}, \\
W^{2} & =\frac{1}{4}\left(z^{\prime}(t)\right)^{-1} z^{\prime \prime}\left(z^{\prime}(t)\right)^{-1} z^{\prime \prime}+\frac{1}{2}\left(z^{\prime}(t)\right)^{-1} z^{\prime \prime} A+\frac{1}{2} A\left(z^{\prime}(t)\right)^{-1} z^{\prime \prime}+A^{2} .
\end{aligned}
$$

Следовательно,

$$
\begin{aligned}
W^{\prime}+W^{2}=- & \frac{1}{2}\left(z^{\prime}(t)\right)^{-1} z^{\prime \prime \prime}+\frac{3}{4}\left[\left(z^{\prime}(t)\right)^{-1} z^{\prime \prime}\right]^{2}-A^{\prime} \\
& +\frac{1}{2}\left(z^{\prime}(t)\right)^{-1} z^{\prime \prime} A+\frac{1}{2} A\left(z^{\prime}(t)\right)^{-1} z^{\prime \prime}+A^{2} .
\end{aligned}
$$

В силу (19) последняя формула дает

$$
W^{\prime}+W^{2}=-\frac{1}{2} S(z)-A^{\prime}+A^{2}-\frac{1}{2}(2 W+2 A) A-\frac{1}{2} A(2 W+2 A)
$$

или

$$
W^{\prime}+W^{2}+W A+A W=-\frac{1}{2} S(z)-A^{\prime}-A^{2} .
$$

Назовем

$$
S(z(t)) \equiv\left[\left(z^{\prime}(t)\right)^{-1} z^{\prime \prime}\right]^{\prime}-\frac{1}{2}\left[\left(z^{\prime}(t)\right)^{-1} z^{\prime \prime}\right]^{2}=2 B(t)-2 A^{\prime}(t)-2 A^{2}
$$

уравнением Швариа для гамильтоновой системы (13) (или, что то же, для уравнения Риккати (17)).

Мы доказали следующее утверждение.

ТеОрема 3. Если $z(\cdot)$ - решение уравнения Швариа $(21)$, то $W(\cdot)$, определяемое формулой (19), есть решение уравнения Риккати (17).

Если $W(\cdot)$ - решение уравнения Риккати (17), то любая функция $z(\cdot)$, полученная при решении линейного относительно $z(\cdot)$ уравнения $(19)$, является решением уравнения Швариа (21).

Как отмечалось в начале статьи, соответствие Сато ассоциирует с КПиерархией систему коммутирующих векторных полей на $\mathrm{GrS}$, которые являются операторным обобщением полей, отвечающих уравнениям Риккати. Но, к сожалению, траектории этих полей не лежат в GrS даже на сколь угодно малом интервале времени. Было бы очень интересно обойти эту трудность и найти правильный аналог теоремы 3 для грассманиана Сато. 
Автор выражает искреннюю благодарность А. Н. Паршину и А. А. Аграчеву, беседы с которыми стимулировали его исследования. Автор также глубоко признателен авторам нижеприведенного списка цитированной литературы, где он почерпнул важные для этой работы идеи.

\section{Список литературы}

[1] M. Sato, Y. Sato, "Soliton equations as dynamical systems on infinite dimensional Grassmann manifold", Lecture Notes Numer. Appl. Anal., 5 (1982), 259-271.

[2] G. Segal, G. Wilson, "Loop groups and equations of the KdV type", Publ. Math. IHES, 80:61 (1985), 301-342.

[3] A. N. Parshin, "Integrable systems and local fields", Comm. Algebra, 29:9 (2001), 4157-4181.

[4] М.И. Зеликин, Однороднъе пространства и уравнение Риккати в вариационном исчислении, Факториал, М., 1998.

[5] M. Mulase, "Solvability of the super KP equations and a generalization of the Birkhoff decomposition", Invent. Math., 92 (1988), 1-46.

[6] А.Н. Паршин, "О кольце формальных псевдодифференциальных операторов", Труды МИАН, 224 (1999), 291-305.

[7] М.И. Зеликин, " $\zeta$-Функция двойного отношения на грассманиане Сато как интегральный инвариант иерархии Кадомцева-Петвиашвили”, Докл. РАН, 389:2 (2003), 159-163.

[8] L. J. Mason, M. A. Singer, N. M. J. Woodhouse, "Tau functions and the twistor theory of integrable systems", J. Geom. Phys., 32 (2000), 397-430.

[9] M. Lauzon, S. Treil, "Common complements of two subspaces of a Hilbert space", J. Funct. Anal., 212:2 (2004), 500-512.

[10] M. Atijah, "Complex analytic connections in fiber bundles", Trans. Amer. Math. Soc., 85 (1957), 181-207.

[11] А.Н. Тюрин, Квантование, классическая и квантовая теория поля и тэтафункиия, Современная математика. Институт компьютерных исследований, М., Ижевск, 2003.

[12] K. Takasaki, "Symmetries and tau function of higher dimensional dispersionless integrable hierarchies", J. Math. Phys., 36:7 (1995), 3574-3607.

М. И. Зеликин (M. I. Zelikin)

Поступила в редакцию

Московский государственный университет

21.03.2005

им. М. В. Ломоносова 